Der vorliegende Artikel schaut in die Zukunft. Das Nationale Forschungsprogramm 74 Gesundheitsversorgung geht uns alle an: Die aus 173 Projektskizzen in einem mehrstufigen Prozess ausgewählten 29 Forschungsprojekte betreffen die ambulante, die stationäre sowie die sektorenübergreifende Versorgung und speziell auch die Versorgung zu Hause. Als Ärztinnen und Ärzte figurieren wir in diversen Rollen in diesem Programm: alle als Leistungserbringer, viele als Teilnehmende an den Studien, einige standespolitisch Aktive als Stakeholder und wenige als Forschende. Insofern ist es lohnend, sich mit dem NFP 74 auseinanderzusetzen.

\title{
Wissenschaftliche Grundlagen für die Optimierung des Gesundheitssystems
}

\section{Milo Puhan}

Prof. Dr., Präsident der Leitungsgruppe NFP 74

\author{
Das Schweizer Gesundheitswesen ist bisher stark auf akute Krankheiten ausge- \\ richtet. Doch es muss den Blick ausweiten und vermehrt auf Zusammenhänge \\ richten. Das Nationale Forschungsprogramm 74 Gesundheitsversorgung soll dafür \\ Lösungen entwickeln.
}

Es ist eine grosse Herausforderung für das Schweizer Gesundheitssystem: In der Schweiz nehmen chronische Krankheiten zu. Sie verursachen den grössten Teil der Krankheitslast und Kosten in unserem Gesundheitswesen, dieses ist aber noch stark auf akute Krankheiten und deren Behandlung ausgerichtet.

Die Gesundheitsversorgung muss deshalb den Fokus neu ausrichten und den Blick auf Zusammenhänge ausweiten: unnötige oder gar schädliche Behandlungen vermeiden, die Zusammenarbeit zwischen verschiedenen Berufsgruppen und Institutionen verbessern, Gesundheitsdaten zusammenführen und den chancengleichen Zugang zur Gesundheitsversorgung sicherstellen.

Dies heisst auch: Für eine gute, nachhaltig gesicherte und «smarte» Gesundheitsversorgung benötigen wir innovative Modelle, eine evidenzbasierte Prävention, Medizin und Pflege, die den Bedürfnissen der Patientinnen und Patienten angemessen Rechnung tragen. Voraussetzung dafür sind wissenschaftliche Grundlagen. Diese will das Nationale Forschungsprogramm (NFP) 74 Gesundheitsversorgung bereitstellen.

Das NFP 74 verfolgt drei Ziele:

- Erkenntnisse gewinnen, wie sich die Struktur der Gesundheitsversorgung und der Zugang dazu verbessern lassen - und damit die Gesundheit der Patientinnen und Patienten,
- längerfristig die Verfügbarkeit, Zugänglichkeit und Verknüpfung von Gesundheitsdaten optimieren

- sowie zum Aufbau einer starken Forschungsgemeinschaft beitragen, die weltweit führende Versorgungsforschung betreibt.

\section{Am Anfang stand eine Initiative der Ärzteschaft}

Das Programm geht zurück auf eine Initiative von Vertreterinnen und Vertretern der Hausarztmedizin und der Inneren Medizin, der Schweizerischen Akademie der Medizinischen Wissenschaften und der Gesundheitspolitik. In der Folge erarbeiteten Mitglieder der heutigen Leitungsgruppe ein Konzept zur Stärkung der Versorgungsforschung, aus welchem das NFP 74 herausgegangen ist. Es wurde 2015 vom Bundesrat genehmigt, ist in diesem Jahr mit 29 Einzelprojekten gestartet und soll bis 2022 dauern; erste Forschungsresultate sind ab 2020 zu erwarten. Das Budget beträgt 20 Millionen Franken.

Die Projekte erforschen Fragen, die für Ärzte von unmittelbarem Interesse sind, und viele der Projekte werden von Ärztinnen und Ärzten durchgeführt und geleitet. Ganz wichtig ist es, dass die Forschungsergebnisse in den Schweizer Kontext gestellt werden, um die Implementierung von Erkenntnissen in der Praxis zu erleichtern. Alle Forschenden des NFP 74 sind aufgefor- 


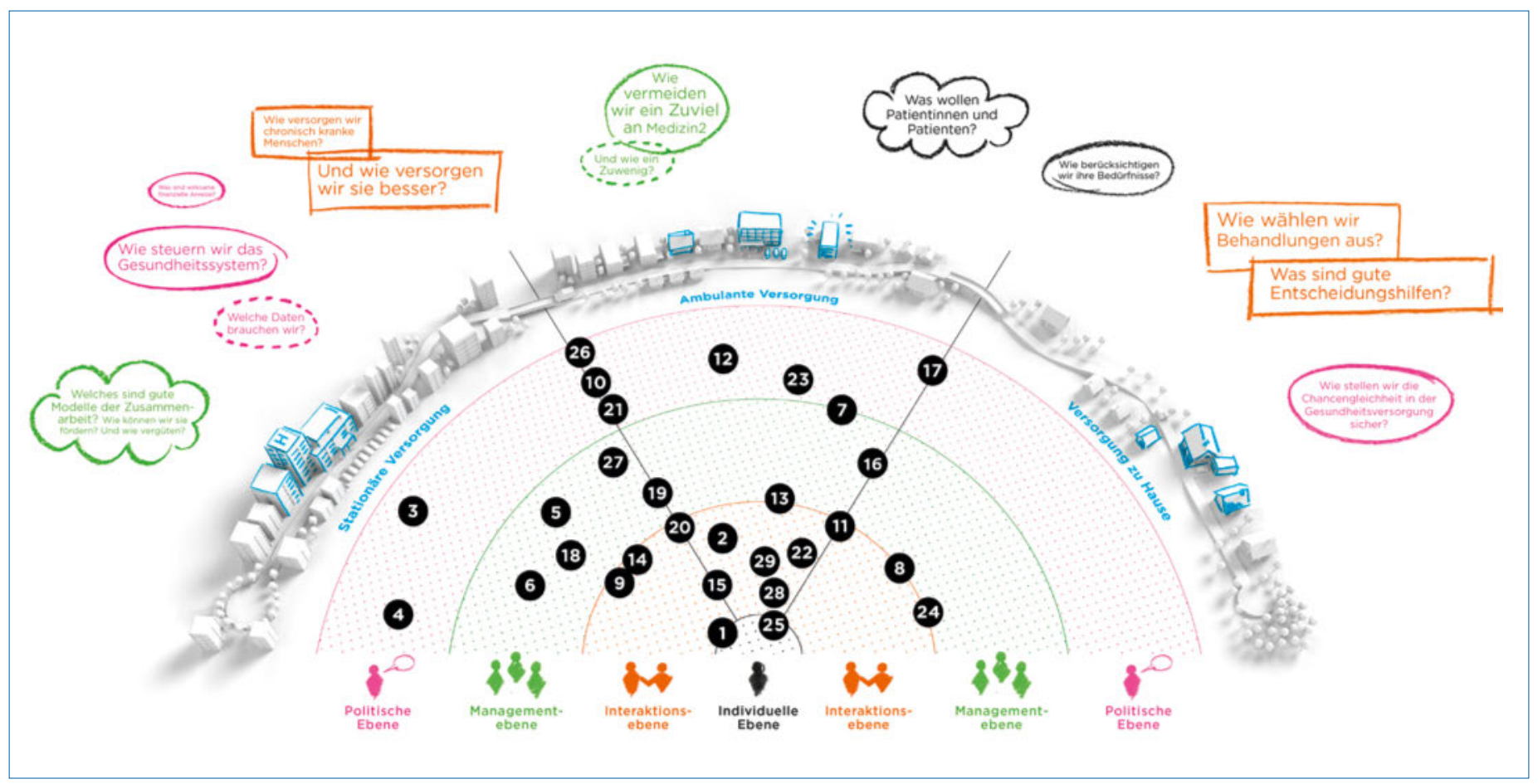

Die 29 Projekte des NFP 74, eingeordnet in die Sektoren und Ebenen der Forschungslandschaft.

\section{Von der SÄZ ausgewählte Projekte des NFP 74}

Prof. Dr. Patrick Bodenmann, Université de Lausanne

Mit Einführung von Case Management die Notfallstationen entlasten

Die Notfallstationen in der Schweiz sind grösstenteils überlastet. Mitverantwortlich dafür ist eine kleine Gruppe von Patientinnen und Patienten, die den Notfall überdurchschnittlich beansprucht. Die Einführung einer Massnahme nach dem Prinzip des Case Managements könnte Abhilfe schaffen.

Prof. Dr. med. Steffen Eychmüller, Universität Bern

Mehr Qualität und weniger Leiden dank besserer Planung und Koordination?

Die meisten Menschen möchten ihr Lebensende selbstbestimmt und im vertrauten Umfeld verbringen. Die Studie untersucht, ob Spitaleinweisungen auch bei einer Verschlechterung der gesundheitlichen Situation vermieden werden können, wenn die Behandlung und Pflege vorausschauend geplant und optimal koordiniert werden.

Prof. Dr. med. Oskar Jenni, Universität Zürich

Die Versorgung von Kindern mit Entwicklungsstörungen im Kanton Zürich

In der Schweiz ist wenig bekannt über Angebot, Nachfrage und Wirksamkeit von Versorgungsleistungen für Kinder mit Entwicklungsauffälligkeiten und -störungen in den ersten Lebensjahren. Das Forschungsprojekt will diese Lücke füllen und analysiert Daten eines zentralen Registers im Kanton Zürich.

\section{Dr. med. Stefan Neuner-Jehle, Universität Zürich}

Optimierte Medikation und Kommunikation bei Spitalaustritt

Die Medikamentenlisten von Patientinnen und Patienten werden immer länger. Dies führt zu unerwünschten Wechselwirkungen und Gesundheitsbeeinträchtigungen. Die systematische Überprüfung der Medikamente bei Spitalaustritt und die standardisierte Kommunikation mit den Hausärztinnen und Hausärzten können hier eine entscheidende Verbesserung bringen.

Prof. Dr. med. Nicolas Rodondi, Universität Bern

Verbesserte Medikation bei chronisch Kranken dank elektronischen Entscheidungshilfen Mehrfachkranke nehmen oft zu viele oder ungeeignete Medikamente ein. Anhand dieser Studie soll ermittelt werden, ob elektronische Entscheidungshilfen für Hausärzte eine Verbesserung der Medikation und der Lebensqualität der Betroffenen herbeiführen. dert, ihren wichtigen Beitrag zu leisten. Der Wissenstransfer ist ein wesentliches Element, und die Forschenden werden darin intensiv unterstützt. Zusätzlich sind Anlässe geplant, wo der Austausch zwischen den Forschenden und Anspruchsgruppen gefördert wird. Den Patienten sollen die Forschungsergebnisse eine bestmögliche Versorgung nach ihren medizinischen Bedürfnissen und Präferenzen bringen. Die Projekte sollen aufzeigen, wo die optimale Balance für eine Versorgung liegt, das heisst, wo man zu wenig und wo man zu viel Medizin macht. Wenn es Ärztinnen und Ärzten sowie Patientinnen und Patienten ermöglicht wird, die bestmögliche Diagnostik und Therapie evidenzbasiert zu wählen, haben die Patientinnen und Patienten den grösstmöglichen Nutzen vom NFP 74 - und davon profitiert auch die Ärzteschaft.

\section{Projekte, in verschiedenen Sektoren und eingeordnet auf verschiedenen Ebenen}

Die Forschungsprojekte untersuchen Fragen zu den drei Versorgungssektoren ambulant, stationär, zu Hause sowie zu den Schnittstellen zwischen diesen Sektoren.

Die Fragen sind auf vier verschiedenen Ebenen angeordnet:

- Auf der individuellen Ebene werden die Präferenzen von unterschiedlichen Patientengruppen er- 
Korrespondenz:

Dr. Kathrin Peter Programmmanagerin NFP 74

Schweizerischer National-

fonds zur Förderung

der wissenschaftlichen

Forschung (SNF)

Wildhainweg 3

CH-3001 Bern

$\mathrm{Tel}+41313082185$

kathrin.peter[at]snf.ch

Manuela Oetterli

Beauftragte für Wissens-

und Technologietransfer

NFP 74

INTERFACE Politikstudien

Forschung Beratung

Seidenhofstrasse 12

CH-6003 Luzern

Tel: +41412260414

oetterli[at]interface-politik-

studien.ch forscht, damit diese besser über den Zugang zu Präventions- und Versorgungsangeboten wie auch zu möglichst guter Behandlung und Betreuung entscheiden können.

- Auf der Interaktionsebene zwischen Gesundheitsfachpersonen und Patientinnen und Patienten werden insbesondere Aspekte der Qualität und der Wahl der Behandlung erforscht. Die Forschungsprojekte untersuchen etwa, wie die Medikation von Menschen mit multiplen chronischen Erkrankungen optimiert werden kann, wie die Präferenzen von Patientinnen und Patienten bei der Behandlungswahl stärker berücksichtigt werden können oder wie Spiritualität in die Behandlung von chronisch kranken Menschen integriert werden kann.

- Auf der Managementebene geht es darum, die Zusammenarbeit zwischen Fachpersonen sowie die Koordination innerhalb und zwischen den verschiedenen Versorgungsbereichen zu optimieren. Beispielsweise wird untersucht, wie durch die interprofessionelle Zusammenarbeit die Dauer des stationären Aufenthalts von Patientinnen und Patienten nicht unnötig verlängert und wie beim Spitalaustritt die Medikation optimiert werden kann. Zudem werden interprofessionelle Modelle in Pflegeheimen und in der palliativen Versorgung entwickelt und getestet.

- Auf der politischen Ebene werden Systemfragen analysiert. Im Hinblick auf eine optimierte Kosteneffizienz werden Anreizsysteme und neue Versor- gungsmodelle geprüft. Zudem werden Möglichkeiten zur Verbesserung und Harmonisierung der Datengrundlagen erforscht. Auch wird der Einfluss von Faktoren wie soziale Gegebenheiten oder medizinische Richtlinien auf die Behandlungswahl und Behandlungsqualität untersucht.

Weitere Informationen - wie ausführliche Beschriebe aller Projekte -, Bestellmöglichkeit für das Programmporträt sowie Anmeldelink für den Newsletter auf www.nfp74.ch

\section{Bildnachweis}

(c) NFP74

\section{Wennberg-Meeting}

Am 12. und 13. April 2018 findet das WennbergMeeting in Zürich statt. Die internationale Konferenz bringt Forschende mit politischen Entscheidungsträgern und Fachleuten aus dem Gesundheitswesen zusammen. Der Fokus liegt auf dem Schweizer Versorgungssystem und seinen regionalen Unterschieden. Organisiert wird die Konferenz vom NFP 74, der Swiss School of Public Health SSPH+ und der Wennberg International Collaborative.

Informationen und Anmeldemöglichkeit auf www.nfp74.ch 